\title{
Some new existence and uniqueness results of solutions to semilinear impulsive fractional integro-differential equations
}

Wen-Xue Zhou ${ }^{1,2^{*}}$, Xu Liu' $^{1}$ and Jian-Gang Zhang ${ }^{1}$

\section{*Correspondence:}

wxzhou2006@126.com

${ }^{1}$ College of Mathematics and

Physics, Lanzhou Jiaotong

University, Lanzhou, 730070, China

${ }^{2}$ School of Mathematical Sciences,

Fudan University, Shanghai, 200433, China

\begin{abstract}
We consider the existence and uniqueness of solutions for the boundary value problem of semilinear impulsive integro-differential equations of fractional order $q \in(1,2]$. Our results are based on the Altman fixed point theorem and a standard fixed point theorem. Two examples are presented to illustrate the main results.
\end{abstract} MSC: $26 \mathrm{~A} 33 ; 34 \mathrm{~B} 15$

Keywords: boundary value problem; impulsive fractional integro-differential equations; Caputo fractional derivative; existence of solutions; fixed point theorem

\section{Introduction}

Boundary value problems for nonlinear fractional differential equations have recently been addressed by several researchers. The interest in the study of differential equations of fractional order lies in the fact that fractional derivatives provide an excellent tool for the description of memory and hereditary properties of various materials and processes [1-11]. For some recent development on the topic, see [12-25] and the references therein. Daftardar-Gejji [12] considered the following system of fractional differential equations:

$$
\begin{aligned}
& D^{\alpha_{i}} u_{i}=f_{i}\left(t, u_{1}, u_{2}, \ldots, u_{n}\right), \quad 0<t<1,0<\alpha_{i}<1,1 \leq i \leq n, \\
& u_{i}(0)=0
\end{aligned}
$$

where $D^{\alpha_{i}}$ denotes Riemann-Liouville derivative of order $\alpha_{i}$. They obtained the existence of a solution by means of the Guo-Krasnosel'skii fixed point theorem and fixed point theorem in a cone.

Babakhani and Daftardar-Gejji [13] considered the following fractional initial value problem:

$$
\left(D^{\alpha_{n}}-\sum_{j=1}^{n-1} p_{j}(x) D^{\alpha_{n-j}}\right) y=f(x, y), \quad y(0)=0, \quad 0 \leq x \leq \lambda, \lambda>0,
$$

where $D^{\alpha_{j}}$ is the standard Riemann-Liouville derivative of order $\alpha_{i}$. They obtained the existence of positive solution by means of the Banach fixed point theorem.

(c) 2015 Zhou et al.; licensee Springer. This is an Open Access article distributed under the terms of the Creative Commons Attribution License (http://creativecommons.org/licenses/by/4.0), which permits unrestricted use, distribution, and reproduction in any medium, provided the original work is properly credited. 
Bai and Lü [14] studied the following two-point boundary value problem of a fractional differential equation:

$$
\begin{aligned}
& D^{\alpha} u(t)+f(t, u(t))=0, \quad 0<t<1,1<\alpha \leq 2, \\
& u(0)=u(1)=0,
\end{aligned}
$$

where $D^{\alpha}$ is the standard Riemann-Liouville fractional derivative. They obtained the existence of positive solutions by means of the Guo-Krasnosel'skii fixed point theorem and the Leggett-Williams fixed point theorem.

Baleanu et al. [15] studied the following boundary value problem of a fractional differential equation:

$$
\begin{aligned}
& D^{\alpha} u(t)+f(t, u(t))=0, \quad 0<\varepsilon<T, T \geq 1,0<\alpha<1, t \in[\varepsilon, T], \\
& u(\eta)=u(T), \quad \eta \in(\varepsilon, t),
\end{aligned}
$$

where $D^{\alpha}$ is the standard Riemann-Liouville fractional derivative. They obtained the existence and uniqueness of positive solutions by means of the fixed point theorem on cones.

Baleanu et al. [16] studied the following initial value problem of a fractional differential equation:

$$
\begin{aligned}
& D^{\alpha} u(t)+D^{\beta} u(t)=f(t, u(t)), \quad 0<t<1,0<\beta<\alpha<1, \\
& u(0)=0,
\end{aligned}
$$

where $D^{\alpha}$ is the standard Riemann-Liouville fractional derivative. They obtained the existence and multiplicity of positive solutions by means of the Guo-Krasnosel'skii fixed point theorem and Leggett-Williams fixed point theorem.

Baleanu et al. [17] studied the existence and uniqueness of a solution for the nonlinear fractional differential equation boundary value problem $D^{\alpha} u(t)=f(t, u(t))$ with a Riemann-Liouville fractional derivative via the different boundary value problems $u(0)=$ $u(T)$, and the three-point boundary condition $u(0)=\beta_{1} u(\eta)$ and $u(T)=\beta_{2} u(\eta)$, where $T>0, t \in I=[0, T], 0<\alpha<1,0<\eta<T, 0<\beta_{1}<\beta_{2}<1$.

By using a fixed point result on ordered metric spaces, Baleanu et al. [18] proved the existence and uniqueness of a solution of the nonlinear fractional differential equation $D^{\alpha} u(t)=f(t, u(t))(t \in I=[0, T], 0<\alpha<1)$ via the periodic boundary condition $u(0)=0$, where $T>0$ and $f: I \times R \rightarrow R$ is a continuous increasing function and ${ }^{c} D^{\alpha}$ denotes the Caputo fractional derivative of order $\alpha$. Also, they solved it by using the anti-periodic boundary conditions $u(0)+u(T)=0$ with $u(0) \leq 0$ and $u(0)+\mu u(T)=0$ with $u(0) \leq 0$ and $\mu>0$ separately.

Zhou and Chu [19] studied the following boundary value problem of a fractional differential equation:

$$
\begin{aligned}
& { }^{c} D^{\alpha} u(t)=f(t, u(t),(K u)(t),(H u)(t)), \quad 0<t<1,1<\alpha \leq 2, \\
& a_{1} u(0)-b_{1} u^{\prime}(0)=d_{1} u\left(\xi_{1}\right), \quad a_{2} u(1)+b_{2} u^{\prime}(1)=d_{2} u\left(\xi_{2}\right),
\end{aligned}
$$


where ${ }^{c} D^{\alpha}$ is the standard Caputo fractional derivative. They obtained the existence and uniqueness of solution by means of the contraction mapping principle and the Krasnoselskii fixed point theorem.

Zhou et al. [20] studied the following boundary value problem of a fractional differential equation:

$$
\begin{aligned}
& { }^{c} D^{\alpha} u(t)=f(t, u(t)), \quad 0 \leq t \leq T, 1<\alpha<2, \\
& u(0)=\lambda_{1} u(T)+\mu_{1}, \quad u^{\prime}(0)=\lambda_{2} u^{\prime}(T)+\mu_{2}, \quad \lambda_{1} \neq 1, \lambda_{2} \neq 1,
\end{aligned}
$$

where ${ }^{c} D^{\alpha}$ is the standard Caputo fractional derivative and $\lambda_{1}, \lambda_{2}, \mu_{1}, \mu_{2} \in \mathbb{R}$. They obtained the existence of solution by means of the Mönch fixed point theorem combined with the technique of measures of weak noncompactness.

Zhou and Liu [21] studied the following boundary value problem of a fractional differential equation:

$$
\begin{aligned}
& { }^{c} D^{\alpha} u(t) \in F(t, u(t)), \quad 0 \leq t \leq T, T>0,1<\alpha \leq 2, \\
& u(0)=\lambda_{1} u(T)+\mu_{1}, \quad u^{\prime}(0)=\lambda_{2} u^{\prime}(T)+\mu_{2},
\end{aligned}
$$

where ${ }^{c} D^{\alpha}$ is the standard Caputo fractional derivative and $\lambda_{1} \neq 1, \lambda_{2} \neq 1, \mu_{1}, \mu_{2} \in \mathbb{R}$. They obtained the existence of solution by means of the Mönch fixed point theorem combined with the technique of measures of weak noncompactness.

Zhou et al. [22] presented some new multiplicity of positive solutions results for nonlinear semi-positone fractional boundary value problem $D^{\alpha} u(t)=p(t) f(t, u(t))-q(t), 0<$ $t<1, u(0)=u(1)=u^{\prime}(1)=0$, where $2<\alpha \leq 3$ is a real number and $D^{\alpha}$ is the standard Riemann-Liouville differentiation.

Zhou et al. [23] studied the following boundary value problem of a fractional differential equation:

$$
\begin{aligned}
& D^{\alpha} u(t)+f(t, u(t))=0, \quad 0<t<1,1<\alpha \leq 2, \\
& u(0)=0, \quad u(1)=a D^{\frac{\alpha-1}{2}} u(\xi),
\end{aligned}
$$

where $\xi \in\left(0, \frac{1}{2}\right], a \in(0,+\infty), a \Gamma(\alpha) \xi^{\frac{\alpha-1}{2}}<\Gamma\left(\frac{\alpha+1}{2}\right)$, and $D^{\alpha}$ is the standard RiemannLiouville fractional derivative. They obtained the uniqueness of positive solution by means of the fixed point theorem in a partially ordered set.

Zhou and Liu [24] discussed the existence of weak solutions for a nonlinear boundary value problem of fractional $q$-difference equations in Banach space. They obtained the uniqueness of positive solution by means of the Mönch fixed point theorem combined with the technique of measures of weak noncompactness.

Zhou et al. [25] studied the following boundary value problem of a fractional differential equation:

$$
\begin{aligned}
& D^{\alpha} u(t)=f(t, u(t)), \quad 0<t<1,1<\alpha \leq 2, \\
& u(0)=u^{\prime}(1)=0,
\end{aligned}
$$


where $D^{\alpha}$ is the standard Riemann-Liouville fractional derivative. They obtained the existence and multiplicity of positive solutions of the nonlinear fractional differential equation boundary value problem by means of the Leray-Schauder nonlinear alternative, a fixed point theorem on cones, and a mixed monotone method. In one word, the nonlinear term $f$ of the above-mentioned boundary value problem does not include $\mathrm{Ku}, \mathrm{Hu}(\mathrm{Ku}, \mathrm{Hu}$ are given functions satisfying some assumptions that will be specified later), and boundary conditions are particularly chosen.

Impulsive differential equations, which provide a natural description of observed evolution processes, are regarded as important mathematical tools for the better understanding of several real world problems in applied sciences. The theory of impulsive differential equations of integer order has found extensive applications in realistic mathematical modeling of a wide variety of practical situations and has emerged as an important area of investigation in recent years. For the general theory and applications of impulsive differential equations, we refer the reader to [26-29]. The impulsive differential equations of fractional order have also attracted considerable attention and a variety of results can be found in [30-39] and the references therein.

Ahmad and Sivasundaram [31] considered the following impulsive fractional differential equations:

$$
\left\{\begin{array}{l}
{ }^{c} D^{q} x(t)=f(t, x(t)), \quad t \in J=[0,1] \backslash\left\{t_{1}, t_{2}, t_{3}, \ldots, t_{p}\right\}, \\
\Delta x\left(t_{k}\right)=I_{k}\left(x\left(t_{k}^{-}\right)\right), \quad \Delta x^{\prime}\left(t_{k}\right)=J_{k}\left(x\left(t_{k}^{-}\right)\right), \quad t_{k} \in(0,1), k=1, \ldots, p, \\
x(0)+x^{\prime}(0)=0, \quad x(1)+x^{\prime}(1)=0,
\end{array}\right.
$$

where ${ }^{c} D^{q}$ is the Caputo fractional derivative. The results are based on the contraction mapping principle and the Krasnoselskii fixed point theorem.

Tian and Bai [33] considered the following impulsive fractional differential equations:

$$
\left\{\begin{array}{l}
{ }^{c} D^{q} u(t)=f(t, u), \quad 0<t<1, t \neq t_{k}, k=1, \ldots, p, 1<q \leq 2, \\
\Delta u\left(t_{k}\right)=I_{k}\left(u\left(t_{k}^{-}\right)\right), \quad \Delta u^{\prime}\left(t_{k}\right)=\bar{I}_{k}\left(u\left(t_{k}^{-}\right)\right), \quad k=1, \ldots, p, \\
u(0)+u^{\prime}(0)=0, \quad u(1)+u^{\prime}(\xi)=0, \quad \xi \in(0,1), \xi \neq t_{k}, k=1, \ldots, p .
\end{array}\right.
$$

The results are based on the contraction mapping principle and the Schauder fixed point theorem.

Zhang and Wang [38] considered the following impulsive fractional differential equations:

$$
\left\{\begin{array}{l}
{ }^{c} D^{q} y(t)=f(t, y), \quad \forall t \in J=[0, T], t \neq t_{k}, k=1, \ldots, m, 1<q \leq 2, \\
\Delta u\left(t_{k}\right)=I_{k}\left(u\left(t_{k}^{-}\right)\right), \quad \Delta u^{\prime}\left(t_{k}\right)=\bar{I}_{k}\left(u\left(t_{k}^{-}\right)\right), \quad k=1, \ldots, p, \\
y(0)=-y(T), \quad y^{\prime}(0)=-y^{\prime}(T) .
\end{array}\right.
$$

The results are based on the Altman fixed point theorem and the Leray-Schauder fixed point theorem.

Zhou and Liu [39] considered the following nonlinear impulsive fractional differential equations:

$$
\left\{\begin{array}{l}
{ }^{c} D^{q} u(t)=f(t, u(t)), \quad 1<q \leq 2, t \in J^{\prime}=J \backslash\left\{t_{1}, t_{2}, \ldots, t_{m}\right\}, \\
\Delta u\left(t_{k}\right)=I_{k}\left(u\left(t_{k}^{-}\right)\right), \quad \Delta u^{\prime}\left(t_{k}\right)=\bar{I}_{k}\left(u\left(t_{k}^{-}\right)\right), \quad k=1, \ldots, m, \\
a u(0)-b u^{\prime}(0)=x_{0}, \quad c u(1)+d u^{\prime}(1)=x_{1} .
\end{array}\right.
$$


The results are based on the nonlinear alternative of Leray-Schauder type and the Krasnoselskii fixed point theorem.

On the other hand, the impulsive boundary value problems for nonlinear fractional differential equations have not been addressed extensively and many aspects of these problems are yet to be explored. For example, we observed that in the above-mentioned work [30-39], the authors all demand that the nonlinear term $f$ is bounded and continuous, and the impulse functions $I_{k}$ and $\bar{I}_{k}$ are bounded, it is easy to see that these conditions are very strong restrictive and difficult to satisfy in applications. Motivated by the abovementioned work [30-39], this article is mainly concerned with the existence and uniqueness of solution for the boundary value problems for the semilinear impulsive fractional integro-differential equations:

$$
\begin{cases}{ }^{c} D^{q} u(t)=\lambda u(t)+f(t, u(t),(K u)(t),(H u)(t)), & 1<q \leq 2, t \in J^{\prime}, \\ \Delta u\left(t_{k}\right)=I_{k}\left(u\left(t_{k}^{-}\right)\right), \quad \Delta u^{\prime}\left(t_{k}\right)=\bar{I}_{k}\left(u\left(t_{k}^{-}\right)\right), & k=1, \ldots, m, \\ a u(0)-b u^{\prime}(0)=x_{0}, & c u(1)+d u^{\prime}(1)=x_{1},\end{cases}
$$

where ${ }^{c} D^{q}$ is the Caputo fractional derivative, $\lambda \geq 0, a \geq 0, b>0, c \geq 0, d>0, \delta=a c+$ $a d+b c \neq 0$, and $x_{0}, x_{1} \in \mathbb{R} . f \in C(I \times \mathbb{R} \times \mathbb{R}, \mathbb{R}), I_{k}, \bar{I}_{k} \in C(\mathbb{R}, \mathbb{R}), J=[0,1], 0=t_{0}<t_{1}<$ $\cdots<t_{m}<t_{m+1}=1, J^{\prime}=J \backslash\left\{t_{1}, t_{2}, \ldots, t_{m}\right\}, \Delta u\left(t_{k}\right)=u\left(t_{k}^{+}\right)-u\left(t_{k}^{-}\right), u\left(t_{k}^{+}\right)=\lim _{h \rightarrow 0^{+}} u\left(t_{k}+h\right)$ and $u\left(t_{k}^{-}\right)=\lim _{h \rightarrow 0^{-}} u\left(t_{k}+h\right)$ represent the right and left limits of $u(t)$ at $t=t_{k}, k=1, \ldots, m$. $\Delta u^{\prime}\left(t_{k}\right)$ has a similar meaning for $u^{\prime}(t)$. Here

$$
\begin{aligned}
& (K u)(t)=\int_{0}^{t} k(t, s) u(s) d s, \quad t \in J, \\
& (H u)(t)=\int_{0}^{1} h(t, s) u(s) d s, \quad t \in J,
\end{aligned}
$$

are integral operators with integral kernels $k \in C\left[D, \mathbb{R}^{+}\right], D=\{(t, s): 0 \leq s \leq t \leq 1\}$, and $h \in C\left[D_{0}, \mathbb{R}^{+}\right], D_{0}=\{(t, s): 0 \leq t, s \leq 1\}, \mathbb{R}^{+}=[0,+\infty)$, where $k^{*}=\sup _{t \in J} \int_{0}^{t}|k(t, s)| d s, h^{*}=$ $\sup _{t \in J} \int_{0}^{1}|h(t, s)| d s$.

Evidently, the problem (1.1) not only includes the boundary value problems mentioned above $[37,39]$ but also extends them to a much wider case. Our main tools are the Altman fixed point theorem and a standard fixed point theorem. Thus, our results are new and generalize some earlier ones.

The remainder of this article is organized as follows. In Section 2, we introduce some preliminary results, including basic definitions of fractional integrals and derivatives, some properties and a fixed point theorem. Section 3 will be devoted to the existence and uniqueness of solutions for boundary value problems of semilinear impulsive integrodifferential equations of fractional order. In the last section, we give two examples to demonstrate the applications.

\section{Preliminaries and lemmas}

Let $J_{0}=\left[0, t_{1}\right], J_{1}=\left(t_{1}, t_{2}\right], \ldots, J_{m-1}=\left(t_{m-1}, t_{m}\right], J_{m}=\left(t_{m}, 1\right]$, and we introduce the spaces: $L^{1}(J, \mathbb{R})$ denotes the Banach space of measurable functions $u: J \mapsto \mathbb{R}$ which are Bochner integrable, equipped with the norm $\|u\|_{L^{1}}:=\int_{J}\|u(t)\| d t, P C(J, \mathbb{R})=\left\{u: J \rightarrow \mathbb{R}: u \in C\left(J_{k}\right)\right.$, $k=0,1, \ldots, m$, and $u\left(t_{k}^{+}\right)$exists, $\left.k=1, \ldots, m\right\}$, is a Banach space with the norm $\|u\|_{P C}:=$ 
$\sup _{t \in J}\|u(t)\|$, and $P C^{1}(J, \mathbb{R})=\left\{u: J \rightarrow \mathbb{R}: u \in C^{1}\left(J_{k}\right), k=0,1, \ldots, m\right.$, and $u\left(t_{k}^{+}\right), u^{\prime}\left(t_{k}^{+}\right)$exist, $k=1, \ldots, m\}$ is a Banach space with the norm $\|u\|_{P C^{1}}:=\max _{t \in J}\left\{\|u\|,\left\|u^{\prime}\right\|\right\}$.

Definition 2.1 (see [1]) The Riemann-Liouville fractional integral of order $r$ for a continuous function $h$ is defined as

$$
I^{r} h(t)=\int_{0}^{t} \frac{(t-s)^{r-1}}{\Gamma(r)} h(s) d s, \quad r>0
$$

provided the integral exists.

Definition 2.2 (see [1]) For an at least $n$-times continuously differentiable function $h$ : $[0, \infty) \rightarrow R$, the Caputo derivative of fractional order $r$ is defined as

$$
{ }^{c} D^{r} h(t)=\frac{1}{\Gamma(n-r)} \int_{0}^{t}(t-s)^{n-r-1} h^{(n)}(s) d s, \quad n-1<r<n, n=[r]+1,
$$

where $[r]$ denotes the integer part of the real number $r$.

Lemma 2.1 (see [1]) Let $r>0, h \in C[0,1] \cap L(0,1)$. Then the differential equation ${ }^{c} D^{r} h(t)=$ 0 has solutions

$$
h(t)=c_{0}+c_{1} t+c_{2} t^{2}+\cdots+c_{n-1} t^{n-1}
$$

where $c_{i} \in \mathbb{R}, i=0,1,2, \ldots, n-1, n=[r]+1$.

Lemma 2.2 (see [1]) Assume that $h \in C[0,1] \cap L(0,1)$ with a derivative of order $r$ that belongs to $C[0,1] \cap L(0,1)$. Then

$$
I_{0+}^{r}{ }^{c} D_{0+}^{r} h(t)=h(t)+c_{0}+c_{1} t+c_{2} t^{2}+\cdots+c_{n-1} t^{n-1},
$$

where $c_{i} \in \mathbb{R}, i=0,1,2, \ldots, n-1, n=[r]+1$.

Lemma 2.3 (see [39]) For a given $h \in C[0,1]$, a function $u$ is a solution of the following impulsive boundary value problem:

$$
\left\{\begin{array}{l}
{ }^{c} D^{q} u(t)=h(t), \quad 1<q \leq 2, t \in J^{\prime}, \\
\Delta u\left(t_{k}\right)=I_{k}\left(u\left(t_{k}^{-}\right)\right), \quad \Delta u^{\prime}\left(t_{k}\right)=\bar{I}_{k}\left(u\left(t_{k}^{-}\right)\right), \quad k=1, \ldots, m, \\
a u(0)-b u^{\prime}(0)=x_{0}, \quad c u(1)+d u^{\prime}(1)=x_{1},
\end{array}\right.
$$

if and only if $u$ is a solution of the impulsive fractional integral equation

$$
u(t)=\left\{\begin{array}{rlr}
\frac{1}{\Gamma(q)} \int_{0}^{t}(t-s)^{q-1} h(s) d s+C_{1}+C_{2} t, & \text { if } t \in J_{0}, \\
\frac{1}{\Gamma(q)} \int_{t_{k}}^{t}(t-s)^{q-1} h(s) d s+\frac{1}{\Gamma(q)} \sum_{i=1}^{k} \int_{t_{i-1}}^{t_{i}}\left(t_{i}-s\right)^{q-1} h(s) d s & \\
& +\frac{1}{\Gamma(q-1)} \sum_{i=1}^{k}\left(t-t_{k}\right) \int_{t_{i-1}}^{t_{i}}\left(t_{i}-s\right)^{q-2} h(s) d s & \\
& +\frac{1}{\Gamma(q-1)} \sum_{i=1}^{k-1}\left(t_{k}-t_{i}\right) \int_{t_{i-1}}^{t_{i}}\left(t_{i}-s\right)^{q-2} h(s) d s & \\
& +\sum_{i=1}^{k} I_{i}\left(y\left(t_{i}^{-}\right)\right)+\sum_{i=1}^{k}\left(t-t_{k}\right) \bar{I}_{i}\left(u\left(t_{i}^{-}\right)\right) & \\
& +\sum_{i=1}^{k-1}\left(t_{k}-t_{i}\right) \bar{I}_{i}\left(u\left(t_{i}^{-}\right)\right)+C_{1}+C_{2} t, & \text { if } t \in J_{k},
\end{array}\right.
$$


where

$$
\begin{aligned}
C_{1}= & -\left\{\sum_{i=1}^{m+1} \frac{b c}{\delta \Gamma(q)} \int_{t_{i-1}}^{t_{i}}\left(t_{i}-s\right)^{q-1} h(s) d s+\sum_{i=1}^{m} \frac{b c\left(1-t_{m}\right)}{\delta \Gamma(q-1)} \int_{t_{i-1}}^{t_{i}}\left(t_{i}-s\right)^{q-2} h(s) d s\right. \\
& +\sum_{i=1}^{m-1} \frac{b c\left(t_{m}-t_{i}\right)}{\delta \Gamma(q-1)} \int_{t_{i-1}}^{t_{i}}\left(t_{i}-s\right)^{q-2} h(s) d s+\sum_{i=1}^{m+1} \frac{b d}{\delta \Gamma(q-1)} \int_{t_{i-1}}^{t_{i}}\left(t_{i}-s\right)^{q-2} h(s) d s \\
& +\sum_{i=1}^{m} \frac{b c}{\delta} I_{i}\left(u\left(t_{i}^{-}\right)\right)+\sum_{i=1}^{m} \frac{b c\left(1-t_{p}\right)}{\delta} \bar{I}_{i}\left(u\left(t_{i}^{-}\right)\right)+\sum_{i=1}^{m-1} \frac{b c\left(t_{p}-t_{i}\right)}{\delta} \bar{I}_{i}\left(u\left(t_{i}^{-}\right)\right) \\
& \left.+\sum_{i=1}^{m} \frac{b d}{\delta} \bar{I}_{i}\left(u\left(t_{i}^{-}\right)\right)+\frac{(b c-\delta) x_{0}-a b x_{1}}{a \delta}\right\}, \\
C_{2}= & -\left\{\sum_{i=1}^{m+1} \frac{a c}{\delta \Gamma(q)} \int_{t_{i-1}}^{t_{i}}\left(t_{i}-s\right)^{q-1} h(s) d s+\sum_{i=1}^{m} \frac{a c\left(1-t_{m}\right)}{\delta \Gamma(q-1)} \int_{t_{i-1}}^{t_{i}}\left(t_{i}-s\right)^{q-2} h(s) d s\right. \\
& +\sum_{i=1}^{m-1} \frac{a c\left(t_{m}-t_{i}\right)}{\delta \Gamma(q-1)} \int_{t_{i-1}}^{t_{i}}\left(t_{i}-s\right)^{q-2} h(s) d s+\sum_{i=1}^{m+1} \frac{a d}{\delta \Gamma(q-1)} \int_{t_{i-1}}^{t_{i}}\left(t_{i}-s\right)^{q-2} h(s) d s \\
& +\sum_{i=1}^{m} \frac{a c}{\delta} I_{i}\left(u\left(t_{i}^{-}\right)\right)+\sum_{i=1}^{m} \frac{a c\left(1-t_{m}\right)}{\delta} \bar{I}_{i}\left(u\left(t_{i}^{-}\right)\right)+\sum_{i=1}^{m-1} \frac{a c\left(t_{m}-t_{i}\right)}{\delta} \bar{I}_{i}\left(u\left(t_{i}^{-}\right)\right) \\
& \left.+\sum_{i=1}^{m} \frac{a d}{\delta} \bar{I}_{i}\left(u\left(t_{i}^{-}\right)\right)+\frac{c x_{0}-a x_{1}}{a \delta}\right\} .
\end{aligned}
$$

We need the following well-known result to prove the existence of solutions for (1.1).

Lemma 2.4 (see [40]) Let E be a Banach space. Assume that $\Omega$ is an open bounded subset of $E$ with $\theta \in \Omega$ and let $T: \bar{\Omega} \rightarrow E$ be a completely continuous operator such that

$$
\|T u\| \leq\|u\|, \quad \forall u \in \partial \Omega .
$$

Then $T$ has a fixed point in $\bar{\Omega}$.

\section{Main results}

Define an operator $\mathcal{A}: P C(J, \mathbb{R}) \rightarrow P C(J, \mathbb{R})$ as

$$
\begin{aligned}
(\mathcal{A} u)(t)= & \int_{t_{k}}^{t} \frac{(t-s)^{q-1}}{\Gamma(q)}[f(s, u(s),(K u)(s),(H u)(s))+\lambda u(s)] d s \\
& +\sum_{i=1}^{k} \int_{t_{i-1}}^{t_{i}} \frac{\left(t_{i}-s\right)^{q-1}}{\Gamma(q)}[f(s, u(s),(K u)(s),(H u)(s))+\lambda u(s)] d s \\
& +\sum_{i=1}^{k}\left(t-t_{k}\right) \int_{t_{i-1}}^{t_{i}} \frac{\left(t_{i}-s\right)^{q-2}}{\Gamma(q-1)}[f(s, u(s),(K u)(s),(H u)(s))+\lambda u(s)] d s \\
& +\sum_{i=1}^{k-1}\left(t_{k}-t_{i}\right) \int_{t_{i-1}}^{t_{i}} \frac{\left(t_{i}-s\right)^{q-2}}{\Gamma(q-1)}[f(s, u(s),(K u)(s),(H u)(s))+\lambda u(s)] d s
\end{aligned}
$$




$$
\begin{aligned}
& +\sum_{i=1}^{k} I_{i}\left(u\left(t_{i}^{-}\right)\right)+\sum_{i=1}^{k}\left(t-t_{k}\right) \bar{I}_{i}\left(u\left(t_{i}^{-}\right)\right) \\
& +\sum_{i=1}^{k-1}\left(t_{k}-t_{i}\right) \bar{I}_{i}\left(u\left(t_{i}^{-}\right)\right)+M_{1}+M_{2} t
\end{aligned}
$$

where

$$
\begin{aligned}
& M_{1}=-\left\{\sum_{i=1}^{m+1} \int_{t_{i-1}}^{t_{i}} \frac{b c\left(t_{i}-s\right)^{q-1}}{\delta \Gamma(q)}[f(s, u(s),(K u)(s),(H u)(s))+\lambda u(s)] d s\right. \\
& +\sum_{i=1}^{m} \int_{t_{i-1}}^{t_{i}} \frac{b c\left(1-t_{m}\right)\left(t_{i}-s\right)^{q-2}}{\delta \Gamma(q-1)}[f(s, u(s),(K u)(s),(H u)(s))+\lambda u(s)] d s \\
& +\sum_{i=1}^{m-1} \int_{t_{i-1}}^{t_{i}} \frac{b c\left(t_{m}-t_{i}\right)\left(t_{i}-s\right)^{q-2}}{\delta \Gamma(q-1)}[f(s, u(s),(K u)(s),(H u)(s))+\lambda u(s)] d s \\
& +\sum_{i=1}^{m+1} \int_{t_{i-1}}^{t_{i}} \frac{b d\left(t_{i}-s\right)^{q-2}}{\delta \Gamma(q-1)}[f(s, u(s),(K u)(s),(H u)(s))+\lambda u(s)] d s \\
& +\sum_{i=1}^{m} \frac{b c}{\delta} I_{i}\left(u\left(t_{i}^{-}\right)\right)+\sum_{i=1}^{m} \frac{b c\left(1-t_{m}\right)}{\delta} \bar{I}_{i}\left(u\left(t_{i}^{-}\right)\right)+\sum_{i=1}^{m-1} \frac{b c\left(t_{m}-t_{i}\right)}{\delta} \bar{I}_{i}\left(u\left(t_{i}^{-}\right)\right) \\
& \left.+\sum_{i=1}^{m} \frac{b d}{\delta} \bar{I}_{i}\left(u\left(t_{i}^{-}\right)\right)+\frac{(b c-\delta) x_{0}-a b x_{1}}{a \delta}\right\} \\
& M_{2}=-\left\{\sum_{i=1}^{m+1} \int_{t_{i-1}}^{t_{i}} \frac{a c\left(t_{i}-s\right)^{q-1}}{\delta \Gamma(q)}[f(s, u(s),(K u)(s),(H u)(s))+\lambda u(s)] d s\right. \\
& +\sum_{i=1}^{m} \int_{t_{i-1}}^{t_{i}} \frac{a c\left(1-t_{m}\right)\left(t_{i}-s\right)^{q-2}}{\delta \Gamma(q-1)}[f(s, u(s),(K u)(s),(H u)(s))+\lambda u(s)] d s \\
& +\sum_{i=1}^{m-1} \int_{t_{i-1}}^{t_{i}} \frac{a c\left(t_{m}-t_{i}\right)\left(t_{i}-s\right)^{q-2}}{\delta \Gamma(q-1)}[f(s, u(s),(K u)(s),(H u)(s))+\lambda u(s)] d s \\
& +\sum_{i=1}^{m+1} \int_{t_{i-1}}^{t_{i}} \frac{\operatorname{ad}\left(t_{i}-s\right)^{q-2}}{\delta \Gamma(q-1)}[f(s, u(s),(K u)(s),(H u)(s))+\lambda u(s)] d s \\
& +\sum_{i=1}^{m} \frac{a c}{\delta} I_{i}\left(u\left(t_{i}^{-}\right)\right)+\sum_{i=1}^{m} \frac{a c\left(1-t_{m}\right)}{\delta} \bar{I}_{i}\left(u\left(t_{i}^{-}\right)\right)+\sum_{i=1}^{m-1} \frac{b c\left(t_{m}-t_{i}\right)}{\delta} \bar{I}_{i}\left(u\left(t_{i}^{-}\right)\right) \\
& \left.+\sum_{i=1}^{m} \frac{a d}{\delta} \bar{I}_{i}\left(u\left(t_{i}^{-}\right)\right)+\frac{c x_{0}-a x_{1}}{a \delta}\right\}
\end{aligned}
$$

Theorem 3.1 Let

$$
\lim _{u \rightarrow 0} \frac{f(t, u, K u, H u)+\lambda u}{u}=0, \quad \lim _{u \rightarrow 0} \frac{I_{k}(u)}{u}=0, \quad \lim _{u \rightarrow 0} \frac{\bar{I}_{k}(u)}{u}=0 .
$$

Then the problem (1.1) has at least one solution on J. 
Proof Consider the operator $\mathcal{A}$ defined by (3.1). Firstly, we show that the operator $\mathcal{A}$ : $P C(J, \mathbb{R}) \rightarrow P C(J, \mathbb{R})$ is continuous and completely continuous. Note that $\mathcal{A}$ is continuous in view of continuity of $f, I_{k}$, and $\bar{I}_{k}$. Let $\Omega \subset P C(J, \mathbb{R})$ be bounded. Then there exist positive constants $L_{i}>0(i=0,1,2,3)$ such that $|u| \leq L_{0},|f(t, u, K u, H u)| \leq L_{1},\left|I_{k}(u)\right| \leq L_{2},\left|\bar{I}_{k}(u)\right| \leq$ $L_{3}, \forall u \in \Omega$. Thus, for any $u \in \Omega$, we get

$$
\begin{aligned}
& \left|M_{1}\right| \leq \sum_{i=1}^{m+1} \int_{t_{i-1}}^{t_{i}} \frac{b c\left(t_{i}-s\right)^{q-1}}{\delta \Gamma(q)}|f(s, u(s),(K u)(s),(H u)(s))+\lambda u(s)| d s \\
& +\sum_{i=1}^{m} \int_{t_{i-1}}^{t_{i}} \frac{b c\left(1-t_{m}\right)\left(t_{i}-s\right)^{q-2}}{\delta \Gamma(q-1)}|f(s, u(s),(K u)(s),(H u)(s))+\lambda u(s)| d s \\
& +\sum_{i=1}^{m-1} \int_{t_{i-1}}^{t_{i}} \frac{b c\left(t_{m}-t_{i}\right)\left(t_{i}-s\right)^{q-2}}{\delta \Gamma(q-1)}|f(s, u(s),(K u)(s),(H u)(s))+\lambda u(s)| d s \\
& +\sum_{i=1}^{m+1} \int_{t_{i-1}}^{t_{i}} \frac{b d\left(t_{i}-s\right)^{q-2}}{\delta \Gamma(q-1)}|f(s, u(s),(K u)(s),(H u)(s))+\lambda u(s)| d s \\
& +\sum_{i=1}^{m} \frac{b c}{\delta}\left|I_{i}\left(u\left(t_{i}\right)\right)\right|+\sum_{i=1}^{m} \frac{b c\left(1-t_{m}\right)}{\delta}\left|\bar{I}_{i}\left(u\left(t_{i}\right)\right)\right|+\sum_{i=1}^{m-1} \frac{b c\left(t_{m}-t_{i}\right)}{\delta}\left|\bar{I}_{i}\left(u\left(t_{i}\right)\right)\right| \\
& +\sum_{i=1}^{m} \frac{b d}{\delta}\left|\bar{I}_{i}\left(u\left(t_{i}\right)\right)\right|+\frac{(c+d)\left|x_{0}\right|+b\left|x_{1}\right|}{\delta} \\
& \leq \sum_{i=1}^{m+1} \int_{t_{i-1}}^{t_{i}} \frac{b c\left(t_{i}-s\right)^{q-1}}{\delta \Gamma(q)}\left(L_{1}+\lambda L_{0}\right) d s+\sum_{i=1}^{m} \frac{b c}{\delta} L_{2} \\
& +\sum_{i=1}^{m} \int_{t_{i-1}}^{t_{i}} \frac{b c\left(1-t_{m}\right)\left(t_{i}-s\right)^{q-2}}{\delta \Gamma(q-1)}\left(L_{1}+\lambda L_{0}\right) d s+\sum_{i=1}^{m} \frac{b c\left(1-t_{m}\right)}{\delta} L_{3} \\
& +\sum_{i=1}^{m-1} \int_{t_{i-1}}^{t_{i}} \frac{b c\left(t_{m}-t_{i}\right)\left(t_{i}-s\right)^{q-2}}{\delta \Gamma(q-1)}\left(L_{1}+\lambda L_{0}\right) d s+\sum_{i=1}^{m-1} \frac{b c\left(t_{m}-t_{i}\right)}{\delta} L_{3} \\
& +\sum_{i=1}^{m+1} \int_{t_{i-1}}^{t_{i}} \frac{b d\left(t_{i}-s\right)^{q-2}}{\delta \Gamma(q-1)}\left(L_{1}+\lambda L_{0}\right) d s+\sum_{i=1}^{m} \frac{b d}{\delta} L_{3}+\frac{(c+d)\left|x_{0}\right|+b\left|x_{1}\right|}{\delta} \\
& \leq\left[\frac{(m+1) b c}{\delta \Gamma(q+1)}+\frac{m b c}{\delta \Gamma(q)}+\frac{(m-1) b c}{\delta \Gamma(q)}+\frac{(m+1) b d}{\delta \Gamma(q)}\right]\left(L_{1}+\lambda L_{0}\right)+\frac{m b c}{\delta} L_{2} \\
& +\left[\frac{m b c}{\delta}+\frac{(m-1) b c}{\delta}+\frac{m b d}{\delta}\right] L_{3}+\frac{(c+d)\left|x_{0}\right|+b\left|x_{1}\right|}{\delta} \text {. }
\end{aligned}
$$

Similarly, we have

$$
\begin{aligned}
\left|M_{2}\right| \leq & \sum_{i=1}^{m+1} \int_{t_{i-1}}^{t_{i}} \frac{a c\left(t_{i}-s\right)^{q-1}}{\delta \Gamma(q)}\left(L_{1}+\lambda L_{0}\right) d s+\sum_{i=1}^{m} \frac{a c}{\delta} L_{2} \\
& +\sum_{i=1}^{m} \int_{t_{i-1}}^{t_{i}} \frac{a c\left(1-t_{m}\right)\left(t_{i}-s\right)^{q-2}}{\delta \Gamma(q-1)}\left(L_{1}+\lambda L_{0}\right) d s+\sum_{i=1}^{m} \frac{a c\left(1-t_{m}\right)}{\delta} L_{3} \\
& +\sum_{i=1}^{m-1} \int_{t_{i-1}}^{t_{i}} \frac{a c\left(t_{m}-t_{i}\right)\left(t_{i}-s\right)^{q-2}}{\delta \Gamma(q-1)}\left(L_{1}+\lambda L_{0}\right) d s+\sum_{i=1}^{m-1} \frac{a c\left(t_{m}-t_{i}\right)}{\delta} L_{3}
\end{aligned}
$$




$$
\begin{aligned}
& +\sum_{i=1}^{m+1} \int_{t_{i-1}}^{t_{i}} \frac{a d\left(t_{i}-s\right)^{q-2}}{\delta \Gamma(q-1)}\left(L_{1}+\lambda L_{0}\right) d s+\sum_{i=1}^{m} \frac{a d}{\delta} L_{3}+\frac{c\left|x_{0}\right|+a\left|x_{1}\right|}{\delta} \\
\leq & {\left[\frac{(m+1) a c}{\delta \Gamma(q+1)}+\frac{m a c}{\delta \Gamma(q)}+\frac{(m-1) a c}{\delta \Gamma(q)}+\frac{(m+1) a d}{\delta \Gamma(q)}\right]\left(L_{1}+\lambda L_{0}\right)+\frac{m a c}{\delta} L_{2} } \\
& +\left[\frac{m a c}{\delta}+\frac{(m-1) a c}{\delta}+\frac{m b d}{\delta}\right] L_{3}+\frac{c\left|x_{0}\right|+a\left|x_{1}\right|}{\delta} .
\end{aligned}
$$

Therefore, we obtain

$$
\begin{aligned}
|A u(t)| \leq & \int_{t_{k}}^{t} \frac{(t-s)^{q-1}}{\Gamma(q)}|f(s, u(s),(K u)(s),(H u)(s))+\lambda u(s)| d s \\
& +\sum_{i=1}^{k} \int_{t_{i-1}}^{t_{i}} \frac{\left(t_{i}-s\right)^{q-1}}{\Gamma(q)}|f(s, u(s),(K u)(s),(H u)(s))+\lambda u(s)| d s \\
& +\sum_{i=1}^{k}\left(t-t_{k}\right) \int_{t_{i-1}}^{t_{i}} \frac{\left(t_{i}-s\right)^{q-2}}{\Gamma(q-1)}|f(s, u(s),(K u)(s),(H u)(s))+\lambda u(s)| d s \\
& +\sum_{i=1}^{k-1}\left(t_{k}-t_{i}\right) \int_{t_{i-1}}^{t_{i}} \frac{\left(t_{i}-s\right)^{q-2}}{\Gamma(q-1)}|f(s, u(s),(K u)(s),(H u)(s))+\lambda u(s)| d s \\
& +\sum_{i=1}^{k}\left|I_{i}\left(u\left(t_{i}^{-}\right)\right)\right|+\sum_{i=1}^{k}\left(t-t_{k}\right)\left|\bar{I}_{i}\left(u\left(t_{i}^{-}\right)\right)\right| \\
& +\sum_{i=1}^{k-1}\left(t_{k}-t_{i}\right)\left|\bar{I}_{i}\left(u\left(t_{i}^{-}\right)\right)\right|+\left|M_{1}\right|+\left|M_{2}\right| \\
\leq & {\left[\int_{t_{k}}^{t} \frac{(t-s)^{q-1}}{\Gamma(q)} d s+\sum_{i=1}^{m} \int_{t_{i-1}}^{t_{i}} \frac{\left(t_{i}-s\right)^{q-1}}{\Gamma(q)} d s\right.} \\
& \left.+\sum_{i=1}^{m} \int_{t_{i-1}}^{t_{i}} \frac{\left(t-t_{k}\right)\left(t_{i}-s\right)^{q-2}}{\Gamma(q-1)} d s+\sum_{i=1}^{m-1} \int_{t_{i-1}}^{t_{i}} \frac{\left(t_{k}-t_{i}\right)\left(t_{i}-s\right)^{q-2}}{\Gamma(q-1)} d s\right]\left(L_{1}+\lambda L_{0}\right) \\
& +\sum_{i=1}^{m} L_{2}+\sum_{i=1}^{m}\left(t-t_{k}\right) L_{3}+\sum_{i=1}^{m-1}\left(t_{k}-t_{i}\right) L_{3}+\left|M_{1}\right|+\left|M_{2}\right| \\
\leq & \frac{(m+1)[c(a+b)+\delta]}{\delta \Gamma(q+1)}\left(L_{1}+\lambda L_{0}\right) \\
& +\frac{(2 m-1)[c(a+b)+\delta]+(m+1)(a+b) d}{\delta}\left(L_{1}+\lambda L_{0}\right) \\
& \frac{(2 c+d)\left|x_{0}\right|+(a+b)\left|x_{1}\right|}{\delta} \\
& \\
&
\end{aligned}
$$

which implies that

$$
\begin{aligned}
\|A u\| \leq & \frac{(m+1)[c(a+b)+\delta]}{\delta \Gamma(q+1)}\left(L_{1}+\lambda L_{0}\right) \\
& +\frac{(2 m-1)[c(a+b)+\delta]+(m+1)(a+b) d}{\delta \Gamma(q)}\left(L_{1}+\lambda L_{0}\right)
\end{aligned}
$$




$$
\begin{aligned}
& +\frac{m[c(a+b)+\delta]}{\delta} L_{2}+\frac{(2 m-1)[c(a+b)+\delta]+m d(a+b)}{\delta} L_{3} \\
& +\frac{(2 c+d)\left|x_{0}\right|+(a+b)\left|x_{1}\right|}{\delta}=: L .
\end{aligned}
$$

On the other hand, for any $t \in J_{k}, 0 \leq k \leq m$, we have

$$
\begin{aligned}
\left|(A u)^{\prime}(t)\right| \leq & \int_{t_{k}}^{t} \frac{(t-s)^{q-2}}{\Gamma(q-1)}|f(s, u(s),(K u)(s),(H u)(s))+\lambda u(s)| d s \\
& +\sum_{i=1}^{k} \int_{t_{i-1}}^{t_{i}} \frac{\left(t_{i}-s\right)^{q-2}}{\Gamma(q-1)}|f(s, u(s),(K u)(s),(H u)(s))+\lambda u(s)| d s \\
& +\sum_{i=1}^{k}\left|\bar{I}_{i}\left(u\left(t_{i}^{-}\right)\right)\right|+\left|M_{2}\right| \\
\leq & \frac{(m+1) a c}{\delta \Gamma(q+1)}\left(L_{1}+\lambda L_{0}\right)+\frac{(2 m-1) a c+(m+1)(a d+\delta)}{\delta \Gamma(q)}\left(L_{1}+\lambda L_{0}\right) \\
& +\frac{m a c}{\delta} L_{2}+\frac{(2 m-1) a c+m(a d+\delta)}{\delta} L_{3}+\frac{c\left|x_{0}\right|+a\left|x_{1}\right|}{\delta}:=\hat{L} .
\end{aligned}
$$

Hence, for $t_{1}, t_{2} \in J_{k}$ with $t_{1}<t_{2}, 0 \leq k \leq m$, we have

$$
\left|(A u)\left(t_{2}\right)-(A u)\left(t_{1}\right)\right| \leq \int_{t_{1}}^{t_{2}}\left|(A u)^{\prime}(s)\right| d s \leq \hat{L}\left(t_{2}-t_{1}\right) .
$$

This implies that $A$ is equicontinuous on all the subintervals $J_{k}, k=0,1,2, \ldots, m$. Thus, by the Arzela-Ascoli theorem, it follows that $\mathcal{A}: P C(J, \mathbb{R}) \rightarrow P C(J, \mathbb{R})$ is completely continuous.

Now, in view of $\lim _{u \rightarrow 0} \frac{f(t, u, K u, H u)+\lambda u}{u}=0, \lim _{u \rightarrow 0} \frac{I_{k}(u)}{u}=0, \lim _{u \rightarrow 0} \frac{\bar{I}_{k}(u)}{u}=0$, there exists a constant $r>0$ such that $|f(t, u, K u, H u)|+\lambda|u| \leq \delta_{1}|u|,\left|I_{k}(u)\right| \leq \delta_{2}|u|$, and $\left|\bar{I}_{k}(u)\right| \leq \delta_{3}|u|$ for $0<|u|<r$, where $\delta_{j}>0(j=1,2,3)$ satisfy

$$
\begin{aligned}
& \frac{(m+1)[c(a+b)+\delta]}{\delta \Gamma(q+1)} \delta_{1}+\frac{(2 m-1)[c(a+b)+\delta]+(m+1)(a+b) d}{\delta \Gamma(q)} \delta_{1}+\frac{m[c(a+b)+\delta]}{\delta} \delta_{2} \\
& +\frac{(2 m-1)[c(a+b)+\delta]+m d(a+b)}{\delta} \delta_{3}+\frac{(2 c+d)\left|x_{0}\right|+(a+b)\left|x_{1}\right|}{\delta} \leq 1 .
\end{aligned}
$$

Let $\Omega=\{u \in P C(J, \mathbb{R}):\|u\|<r\}$ and take $u \in P C(J, \mathbb{R})$ such that $\|u\|=r$ so that $u \in \partial \Omega$. Then, by the process used to obtain (3.7), we have

$$
\begin{aligned}
|A u(t)| \leq & \left\{\frac{(m+1)[c(a+b)+\delta]}{\delta \Gamma(q+1)} \delta_{1}+\frac{(2 m-1)[c(a+b)+\delta]+(m+1)(a+b) d}{\delta \Gamma(q)} \delta_{1}\right. \\
& +\frac{m[c(a+b)+\delta]}{\delta} \delta_{2}+\frac{(2 m-1)[c(a+b)+\delta]+m d(a+b)}{\delta} \delta_{3} \\
& \left.+\frac{(2 c+d)\left|x_{0}\right|+(a+b)\left|x_{1}\right|}{\delta}\right\}\|u\|,
\end{aligned}
$$

which implies that $\|A u\| \leq\|u\|, u \in \partial \Omega$. Therefore, by Lemma 2.4, the operator $A$ has at least one fixed point, which in turn implies that the problem (1.1) has at least one solution $u \in \bar{\Omega}$. This completes the proof. 
Theorem 3.2 Assume that (H1) there exist nonnegative constants $\gamma_{i}(i=1,2,3,4,5)$ such that $|f(t, u, K u, H u)-f(t, \bar{u}, K \bar{u}, H \bar{u})| \leq \gamma_{1}|u-\bar{u}|+\gamma_{2}|K u-K \bar{u}|+\gamma_{3}|H u-H \bar{u}|, t \in J, \mid I_{k}(u)-$ $I_{k}(\bar{u})\left|\leq \gamma_{4}\right| u-\bar{u}|,| \bar{I}_{k}(u)-\bar{I}_{k}(\bar{u})\left|\leq \gamma_{5}\right| u-\bar{u} \mid$, for $t \in J, u, \bar{u} \in \mathbb{R}$ and $k=1,2, \ldots, m$. Then the problem (1.1) has a unique solution if

$$
\begin{aligned}
\Lambda= & \frac{(m+1)[c(a+b)+\delta]}{\delta \Gamma(q+1)}\left(\gamma_{1}+\gamma_{2} k^{*}+\gamma_{3} h^{*}+\lambda\right) \\
& +\frac{(2 m-1)[c(a+b)+\delta]+m d(a+b)}{\delta} \gamma_{5}+\frac{m[c(a+b)+\delta]}{\delta} \gamma_{4} \\
& +\frac{(2 m-1)[c(a+b)+\delta]+(m+1)(a+b) d}{\delta \Gamma(q)}\left(\gamma_{1}+\gamma_{2} k^{*}+\gamma_{3} h^{*}+\lambda\right)<1 .
\end{aligned}
$$

Proof For $u, \bar{u} \in C(J, \mathbb{R})$, it follows by the condition (H1) that

$$
\begin{aligned}
& |f(s, u(s), K u(s), H u(s))-f(s, \bar{u}(s), K \bar{u}(s), H \bar{u}(s))| \\
& \leq \gamma_{1}|u(s)-\bar{u}(s)|+\gamma_{2} \int_{0}^{s} k(s, \tau)|u(\tau)-\bar{u}(\tau)| d \tau \\
& \quad+\gamma_{3} \int_{0}^{1} h(s, \tau)|u(\tau)-\bar{u}(\tau)| d \tau \\
& \leq\left\{\gamma_{1}+\gamma_{2} k^{*}+\gamma_{3} h^{*}\right\}\|u-\bar{u}\| .
\end{aligned}
$$

In view of the estimate (3.10), we obtain

$$
\begin{aligned}
\mid A u(t) & -A \bar{u}(t) \mid \\
\leq & \int_{t_{k}}^{t} \frac{(t-s)^{q-1}}{\Gamma(q)}\left(\gamma_{1}+\gamma_{2} k^{*}+\gamma_{3} h^{*}+\lambda\right)\|u-\bar{u}\| d s \\
& +\sum_{i=1}^{k} \int_{t_{i-1}}^{t_{i}} \frac{\left(t_{i}-s\right)^{q-1}}{\Gamma(q)}\left(\gamma_{1}+\gamma_{2} k^{*}+\gamma_{3} h^{*}+\lambda\right)\|u-\bar{u}\| d s \\
& +\sum_{i=1}^{k}\left(t-t_{k}\right) \int_{t_{i-1}}^{t_{i}} \frac{\left(t_{i}-s\right)^{q-2}}{\Gamma(q-1)}\left(\gamma_{1}+\gamma_{2} k^{*}+\gamma_{3} h^{*}+\lambda\right)\|u-\bar{u}\| d s \\
& +\sum_{i=1}^{k-1}\left(t_{k}-t_{i}\right) \int_{t_{i-1}}^{t_{i}} \frac{\left(t_{i}-s\right)^{q-2}}{\Gamma(q-1)}\left(\gamma_{1}+\gamma_{2} k^{*}+\gamma_{3} h^{*}+\lambda\right)\|u-\bar{u}\| d s \\
& +\sum_{i=1}^{k}\left|I_{i}\left(u\left(t_{i}^{-}\right)\right)-I_{i}\left(\bar{u}\left(t_{i}^{-}\right)\right)\right|+\sum_{i=1}^{k}\left(t-t_{k}\right)\left|\bar{I}_{i}\left(u\left(t_{i}^{-}\right)\right)-\bar{I}_{i}\left(\bar{u}\left(t_{i}^{-}\right)\right)\right| \\
& +\sum_{i=1}^{k-1}\left(t_{k}-t_{i}\right)\left|\bar{I}_{i}\left(u\left(t_{i}^{-}\right)\right)-\bar{I}_{i}\left(\bar{u}\left(t_{i}^{-}\right)\right)\right| \\
& +\sum_{i=1}^{m+1} \int_{t_{i-1}}^{t_{i}} \frac{b c\left(t_{i}-s\right)^{q-1}}{\delta \Gamma(q)}\left(\gamma_{1}+\gamma_{2} k^{*}+\gamma_{3} h^{*}+\lambda\right)\|u-\bar{u}\| d s \\
& +\sum_{i=1}^{m} \int_{t_{i-1}}^{t_{i}} \frac{b c\left(1-t_{m}\right)\left(t_{i}-s\right)^{q-2}}{\delta \Gamma(q-1)}\left(\gamma_{1}+\gamma_{2} k^{*}+\gamma_{3} h^{*}+\lambda\right)\|u-\bar{u}\| d s \\
& +\sum_{i=1}^{m-1} \int_{t_{i-1}}^{t_{i}} \frac{b c\left(t_{m}-t_{i}\right)\left(t_{i}-s\right)^{q-2}}{\delta \Gamma(q-1)}\left(\gamma_{1}+\gamma_{2} k^{*}+\gamma_{3} h^{*}+\lambda\right)\|u-\bar{u}\| d s
\end{aligned}
$$




$$
\begin{aligned}
& +\sum_{i=1}^{m+1} \int_{t_{i-1}}^{t_{i}} \frac{b d\left(t_{i}-s\right)^{q-2}}{\delta \Gamma(q-1)}\left(\gamma_{1}+\gamma_{2} k^{*}+\gamma_{3} h^{*}+\lambda\right)\|u-\bar{u}\| d s \\
& +\sum_{i=1}^{m} \frac{b c}{\delta}\left|I_{i}\left(u\left(t_{i}\right)\right)-I_{i}\left(\bar{u}\left(t_{i}\right)\right)\right|+\sum_{i=1}^{m} \frac{b c\left(1-t_{m}\right)}{\delta}\left|\bar{I}_{i}\left(u\left(t_{i}\right)\right)-\bar{I}_{i}\left(\bar{u}\left(t_{i}\right)\right)\right| \\
& +\sum_{i=1}^{m-1} \frac{b c\left(t_{m}-t_{i}\right)}{\delta}\left|\bar{I}_{i}\left(u\left(t_{i}\right)\right)-\bar{I}_{i}\left(\bar{u}\left(t_{i}\right)\right)\right|+\sum_{i=1}^{m} \frac{b d}{\delta}\left|\bar{I}_{i}\left(u\left(t_{i}\right)\right)-\bar{I}_{i}\left(\bar{u}\left(t_{i}\right)\right)\right| \\
& +\sum_{i=1}^{m+1} \int_{t_{i-1}}^{t_{i}} \frac{a c\left(t_{i}-s\right)^{q-1}}{\delta \Gamma(q)}\left(\gamma_{1}+\gamma_{2} k^{*}+\gamma_{3} h^{*}+\lambda\right)\|u-\bar{u}\| d s \\
& +\sum_{i=1}^{m} \int_{t_{i-1}}^{t_{i}} \frac{a c\left(1-t_{m}\right)\left(t_{i}-s\right)^{q-2}}{\delta \Gamma(q-1)}\left(\gamma_{1}+\gamma_{2} k^{*}+\gamma_{3} h^{*}+\lambda\right)\|u-\bar{u}\| d s \\
& +\sum_{i=1}^{m-1} \int_{t_{i-1}}^{t_{i}} \frac{a c\left(t_{m}-t_{i}\right)\left(t_{i}-s\right)^{q-2}}{\delta \Gamma(q-1)}\left(\gamma_{1}+\gamma_{2} k^{*}+\gamma_{3} h^{*}+\lambda\right)\|u-\bar{u}\| d s \\
& +\sum_{i=1}^{m+1} \int_{t_{i-1}}^{t_{i}} \frac{a d\left(t_{i}-s\right)^{q-2}}{\delta \Gamma(q-1)}\left(\gamma_{1}+\gamma_{2} k^{*}+\gamma_{3} h^{*}+\lambda\right)\|u-\bar{u}\| d s \\
& +\sum_{i=1}^{m} \frac{a c}{\delta}\left|I_{i}\left(u\left(t_{i}^{-}\right)\right)-I_{i}\left(\bar{u}\left(t_{i}^{-}\right)\right)\right|+\sum_{i=1}^{m} \frac{a c\left(1-t_{m}\right)}{\delta}\left|\bar{I}_{i}\left(u\left(t_{i}^{-}\right)\right)-\bar{I}_{i}\left(\bar{u}\left(t_{i}^{-}\right)\right)\right| \\
\leq & +\|u-\bar{u}\|, \\
& +\sum_{i=1}^{m-1} \frac{a c\left(t_{m}-t_{i}\right)}{\delta}\left|\bar{I}_{i}\left(u\left(t_{i}^{-}\right)\right)-\bar{I}_{i}\left(\bar{u}\left(t_{i}^{-}\right)\right)\right|+\sum_{i=1}^{m} \frac{a d}{\delta}\left|\bar{I}_{i}\left(u\left(t_{i}^{-}\right)\right)-\bar{I}_{i}\left(\bar{u}\left(t_{i}^{-}\right)\right)\right| \\
\leq & \frac{(m+1)[c(a+b)+\delta]}{\delta \Gamma(q+1)}\left(\gamma_{1}+\gamma_{2} k^{*}+\gamma_{3} h^{*}+\lambda\right) \\
& \frac{(2 m-1)[c(a+b)+\delta]+m d(a+b)}{\delta} \gamma_{5}+\frac{m[c(a+b)+\delta]}{\delta} \gamma_{4} \\
&
\end{aligned}
$$

where $\Lambda$ is given by (3.9). Thus, $\|A u-A \bar{u}\| \leq \Lambda\|u-\bar{u}\|$. As $\Lambda<1, A$ is a contraction operator. Hence, by the contraction mapping principle, the problem (1.1) has a unique solution. The proof is complete.

Remark 3.1 The problem (1.1) not only includes the boundary value problems mentioned above [37, 39], but it also extends them to a much wider case. Thus, our results are new and generalize some earlier ones.

In the sequel we present two examples which illustrate Theorem 3.1 and Theorem 3.2.

\section{Examples}

Example 4.1 Consider the following boundary value problem:

$$
\left\{\begin{array}{l}
{ }^{c} D_{0+}^{q} u(t)=2 \ln \left(1+u^{2}\right)-\cos u+1, \quad 0<t<1, t \neq t_{1}, 0<t_{1}<1 \\
\Delta u\left(t_{1}\right)=e^{u^{3}\left(t_{1}\right)}-1, \quad \Delta u^{\prime}\left(t_{1}\right)=\left(1+u^{2}\left(t_{1}\right)\right)^{\frac{1}{3}}-1 \\
u(0)-u^{\prime}(0)=0.03, \quad u(1)+u^{\prime}(1)=0.06
\end{array}\right.
$$


where

$$
\begin{aligned}
& f(t, u)=2 \ln \left(1+u^{2}\right)-\cos u+1, \\
& I_{1}(u)=e^{u^{3}}-1, \quad \bar{I}_{1}(u)=\left(1+u^{2}\right)^{\frac{1}{3}}-1 .
\end{aligned}
$$

Here $q=\frac{3}{2}, \lambda=0, a=1, b=1, c=1, d=1, m=1, \delta=a c+a d+b c=3, k(t, s)=0, h(t, s)=0$, $x_{0}=0.03, x_{1}=0.06$. Clearly

$$
\begin{aligned}
& \lim _{u \rightarrow 0} \frac{f(t, u)}{u}=\lim _{u \rightarrow 0} \frac{2 \ln \left(1+u^{2}\right)-\cos u+1}{u}=\lim _{u \rightarrow 0} \frac{2 \ln \left(1+u^{2}\right)}{u}+\lim _{u \rightarrow 0} \frac{-\cos u+1}{u}=0, \\
& \lim _{u \rightarrow 0} \frac{I_{1}(u)}{u}=\lim _{u \rightarrow 0} \frac{e^{u^{3}}-1}{u}=\lim _{u \rightarrow 0} 3 u^{2}=0, \\
& \lim _{u \rightarrow 0} \frac{\bar{I}_{1}(u)}{u}=\lim _{u \rightarrow 0} \frac{\left(1+u^{2}\right)^{\frac{1}{3}}-1}{u}=\lim _{u \rightarrow 0} \frac{u}{3}=0,
\end{aligned}
$$

Furthermore, in this case, $\delta_{i}(i=1,2,3)$ satisfy the inequality

$$
\begin{aligned}
& \frac{(m+1)[c(a+b)+\delta]}{\delta \Gamma(q+1)} \delta_{1}+\frac{(2 m-1)[c(a+b)+\delta]+(m+1)(a+b) d}{\delta \Gamma(q)} \delta_{1}+\frac{m[c(a+b)+\delta]}{\delta} \delta_{2} \\
& +\frac{(2 m-1)[c(a+b)+\delta]+m d(a+b)}{\delta} \delta_{3}+\frac{(2 c+d)\left|x_{0}\right|+(a+b)\left|x_{1}\right|}{\delta} \leq 1 .
\end{aligned}
$$

Thus, all the assumptions of Theorem 3.1 are satisfied. Hence, by the conclusion of Theorem 3.1, the impulsive fractional boundary value problem (4.1) has at least one solution.

Example 4.2 Consider the following boundary value problem:

$$
\left\{\begin{aligned}
&{ }^{c} D_{0+}^{q} u(t)=\frac{1}{40} u(t)+\frac{\sin t}{(t+4)^{2}} \frac{|u(t)|}{1+|u(t)|}+\frac{1}{25} \int_{0}^{t} \frac{e^{-(s-t)}}{5} u(s) d s \\
&+\frac{1}{25} \int_{0}^{1} \frac{e^{-(s-t) / 2}}{5} u(s) d s, \quad 0<t<1, t \neq \frac{3}{5}, \\
& \Delta u\left(\frac{3}{5}\right)=\frac{1}{80} \frac{\left|u\left(\frac{3}{5}\right)\right|}{16+\left|u\left(\frac{3}{5}\right)\right|}, \Delta u^{\prime}\left(\frac{3}{5}\right)=\frac{1}{300} \frac{\left|u\left(\frac{3}{5}\right)\right|}{25+\left|u\left(\frac{3}{5}\right)\right|}, \\
& u(0)-u^{\prime}(0)=0.03, u(1)+u^{\prime}(1)=0.06,
\end{aligned}\right.
$$

where

$$
\begin{aligned}
& f(t, u(t), K u(t), H u(t)) \\
& \quad=\frac{\sin t}{(t+4)^{2}} \frac{|u(t)|}{1+|u(t)|}+\frac{1}{25} \int_{0}^{t} \frac{e^{-(s-t)}}{5} u(s) d s+\frac{1}{25} \int_{0}^{1} \frac{e^{-(s-t) / 2}}{5} u(s) d s,
\end{aligned}
$$

$q=\frac{3}{2}, \lambda=\frac{1}{40}, a=1, b=1, c=1, d=1, m=1, \delta=a c+a d+b c=3, k(t, s)=\frac{e^{-(s-t)}}{5}, h(t, s)=$ $\frac{e^{-(s-t) / 2}}{5}, x_{0}=0.03, x_{1}=0.06$. Clearly $\gamma_{1}=\frac{1}{16}, \gamma_{2}=\frac{1}{25}, \gamma_{3}=\frac{1}{25}, \gamma_{4}=\frac{1}{80}, \gamma_{5}=\frac{1}{300}, k^{*}=\frac{e-1}{5}$, $h^{*}=\frac{2(\sqrt{e}-1)}{5}$.

Moreover, we have

$$
\begin{aligned}
\Lambda= & \frac{(m+1)[c(a+b)+\delta]}{\delta \Gamma(q+1)}\left(\gamma_{1}+\gamma_{2} k^{*}+\gamma_{3} h^{*}+\lambda\right) \\
& +\frac{(2 m-1)[c(a+b)+\delta]+m d(a+b)}{\delta} \gamma_{5}+\frac{m[c(a+b)+\delta]}{\delta} \gamma_{4}
\end{aligned}
$$




$$
\begin{aligned}
& +\frac{(2 m-1)[c(a+b)+\delta]+(m+1)(a+b) d}{\delta \Gamma(q)}\left(\gamma_{1}+\gamma_{2} k^{*}+\gamma_{3} h^{*}+\lambda\right) \\
\approx & 0.68638575<1 .
\end{aligned}
$$

Thus, all the assumptions of Theorem 3.2 are satisfied. Hence, by the conclusion of Theorem 3.2, the impulsive fractional boundary value problem (4.2) has a unique solution on $J$.

\section{Conclusions}

In this paper, the boundary value problem of semilinear impulsive integro-differential equations of fractional order $q \in(1,2]$ have been investigated. Based on the Altman fixed point theorem and a standard fixed point theorem, the existence and uniqueness of solutions for the boundary value problem of semilinear impulsive integro-differential equations of fractional order $q \in(1,2]$ are presented. Two examples are presented to demonstrate the effectiveness and feasibility of the proposed scheme.

\section{Competing interests}

The authors declare that they have no competing interests.

\section{Authors' contributions}

All authors contributed equally to the manuscript. All authors read and approved the final manuscript.

\section{Acknowledgements}

This research was supported by the National Natural Science Foundation of China (11161027, 11262009, and 11226132), the Scientific Research Projects in Colleges and Universities of Gansu Province of China (2013A-043), the Fundamental Research Funds for the Gansu Universities (213061 and 212084), the Youth Science Foundation of Lanzhou Jiaotong University (2012019), and the Natural Science Foundation of Gansu Province of China (1308RJZA113 and 145RJZA195). The authors are thankful to the referees for their careful reading of the manuscript and insightful comments.

Received: 30 October 2014 Accepted: 13 January 2015 Published online: 11 February 2015

\section{References}

1. Kilbas, AA, Srivastava, HM, Trujillo, JJ: Theory and Applications of Fractional Differential Equations. Elsevier, Amsterdam (2006)

2. Miller, KS, Ross, B: An Introduction to the Fractional Calculus and Differential Equations. Wiley, New York (1993)

3. Oldham, KB, Spanier, J: The Fractional Calculus. Academic Press, New York (1974)

4. Podlubny, I: Fractional Differential Equations. Academic Press, San Diego (1999)

5. Samko, SG, Kilbas, AA, Marichev, Ol: Fractional Integrals and Derivatives: Theory and Applications. Gordon \& Breach, Yverdon (1993)

6. Choi, J, Agarval, P: Certain transform fractional integral formulas for the generalized Gauss hypergeometric functions. Abstr. Appl. Anal. 2014, Article ID 735946 (2014)

7. Baleanu, D, Agarwal, P: On generalized fractional integral operators and the generalized Gauss hypergeometric functions. Abstr. Appl. Anal. 2014, Article ID 630840 (2014)

8. Baleanu, D, Agarwal, P: Certain inequalities involving the fractional q-integral operators. Abstr. Appl. Anal. 2014, Article ID 371274 (2014)

9. Choi, J, Agarwal, P: Some new Saigo type fractional integral inequalities and their $q$-analogues. Abstr. Appl. Anal. 2014, Article ID 579260 (2014)

10. Agarwal, P, Jain, S: Further results on fractional calculus of Srivastava polynomials. Bull. Math. Anal. Appl. 3(2), 167-174 (2011)

11. Agarwal, P: Further results on fractional calculus of Saigo operator. Appl. Appl. Math. 7(2), 585-594 (2012)

12. Daftardar-Gejji, V: Positive solutions of a system of non-autonomous nonlinear fractional differential equations. J. Math. Anal. Appl. 302(1), 56-64 (2005)

13. Babakhani, A, Daftardar-Gejji, V: Existence of positive solutions for multi-term non-autonomous fractional differential equations with polynomial coefficients. Electron. J. Differ. Equ. 2006, 129 (2006)

14. Bai, ZB, Lü, H: Positive solutions for boundary value problem of nonlinear fractional differential equation. J. Math. Anal. Appl. 311(2), 495-505 (2005)

15. Baleanu, D, Agarwal, RP, Mohammadi, H, Rezapour, S: Some existence results for a nonlinear fractional differential equation on partially ordered Banach spaces. Bound. Value Probl. 2013, 112 (2013)

16. Baleanu, D, Mohammadi, H, Rezapour, S: Positive solutions of a boundary value problem for nonlinear fractional differential equations. Abstr. Appl. Anal. 2012, Article ID 837437 (2012)

17. Baleanu, D, Mohammadi, H, Rezapour, S: Some existence results on nonlinear fractional differential equations. Philos. Trans. R. Soc. Lond. A 371, 20120144 (2013)

18. Baleanu, D, Mohammadi, H, Rezapour, S: On a nonlinear fractional differential equation on partially ordered metric spaces. Adv. Differ. Equ. 2013, 83 (2013) 
19. Zhou, W-X, Chu, Y-D: Existence of solutions for fractional differential equations with multi-point boundary conditions. Commun. Nonlinear Sci. Numer. Simul. 17(3), 1142-1148 (2012)

20. Zhou, W-X, Chang, Y-X, Liu, H-Z: Weak solutions for nonlinear fractional differential equations in Banach spaces. Discrete Dyn. Nat. Soc. 2012, Article ID 527969 (2012)

21. Zhou, W-X, Liu, H-Z: Existence of weak solutions for nonlinear fractional differential inclusion with non-separated boundary conditions. J. Appl. Math. 2012, Article ID 530624 (2012)

22. Zhou, W-X, Peng, J-G, Chu, Y-D: Multiple positive solutions for nonlinear semipositone fractional differential equations. Discrete Dyn. Nat. Soc. 2012, Article ID 850871 (2012)

23. Zhou, W-X, Chu, Y-D, Baleanu, D: Uniqueness and existence of positive solutions for a multi-point boundary value problem of singular fractional differential equations. Adv. Differ. Equ. 2013, 114 (2013)

24. Zhou, W-X, Liu, H-Z: Existence solutions for boundary value problem of nonlinear fractional q-difference equations. Adv. Differ. Equ. 2013, 113 (2013)

25. Zhou, W-X, Zhang, J-G, Li, J-M: Existence of multiple positive solutions for singular boundary value problems of nonlinear fractional differential equations. Adv. Differ. Equ. 2014, 97 (2014)

26. Bainov, DD, Simeonov, PS: Systems with Impulsive Effect. Ellis Horwood, Chichester (1989)

27. Benchohra, M, Henderson, J, Ntouyas, S: Impulsive Differential Equations and Inclusions. Hindawi Publishing Corporation, New York (2006)

28. Lakshmikantham, V, Bainov, DD, Simeonov, PS: Theory of Impulsive Differential Equations. World Scientific, Singapore (1989)

29. Samoilenko, AM, Perestyuk, NA: Impulsive Differential Equations. World Scientific, Singapore (1995)

30. Agarwal, RP, Benchohra, M, Hamani, S: A survey on existence results for boundary value problems of nonlinear fractional differential equations and inclusions. Acta Appl. Math. 109(3), 973-1033 (2010)

31. Ahmad, B, Sivasundaram, S: Existence results for nonlinear impulsive hybrid boundary value problems involving fractional differential equations. Nonlinear Anal. Hybrid Syst. 3(3), 251-258 (2009)

32. Ahmad, B, Nieto, JJ: Existence of solutions for impulsive anti-periodic boundary value problems of fractional order. Taiwan. J. Math. 15(3), 981-993 (2011)

33. Tian, YS, Bai, Z: Existence results for the three-point impulsive boundary value problem involving fractional differential equations. Comput. Math. Appl. 59(8), 2601-2609 (2010)

34. Henderson, J, Ouahab, A: Impulsive differential inclusions with fractional order. Comput. Math. Appl. 59(3), 1191-1226 (2010)

35. Wang, GT, Ahmad, B, Zhang, L: Impulsive anti-periodic boundary value problem for nonlinear differential equations of fractional order. Nonlinear Anal. 74(3), $792-804$ (2011)

36. Wang, GT, Ahmad, B, Zhang, L: Some existence results for impulsive nonlinear fractional differential equations with mixed boundary conditions. Comput. Math. Appl. 62(3), 1389-1397 (2011)

37. Wang, XH: Impulsive boundary value problem for nonlinear differential equations of fractional order. Comput. Math. Appl. 62(5), 2383-2391 (2011)

38. Zhang, LH, Wang, G: Existence of solutions for nonlinear fractional differential equations with impulses and anti-periodic boundary conditions. Electron. J. Qual. Theory Differ. Equ. 2011, 7 (2011)

39. Zhou, W-X, Liu, X: Existence of solution to a class of boundary value problem for impulsive fractional differential equations. Adv. Differ. Equ. 2014, 12 (2014)

40. Sun, JX: Nonlinear Functional Analysis and Its Application. Science Press, Beijing (2008)

\section{Submit your manuscript to a SpringerOpen ${ }^{\circ}$ journal and benefit from:}

- Convenient online submission

Rigorous peer review

- Immediate publication on acceptance

- Open access: articles freely available online

- High visibility within the field

- Retaining the copyright to your article 